\section{Secondary Fibromyalgia}

The concept of secondary fibromyalgia (FM) has garnered increasing interest over the last decade. This has resulted from the realization that many common rheumatic disorders have an FM comorbidity that influences, or should influence, optimal management ${ }^{1,2}$. In this edition of The Journal, Wolfe and colleagues reconsider the issue of primary and secondary FM, asking whether they are equivalent ${ }^{3}$. Currently, there is no universally agreed-upon definition. Dr. Kahler Hench, the originator of the term fibromyalgia, provided this definition: "Fibrositis is considered primary when there is no associated underlying disorder and secondary when it occurs in patients with underlying rheumatic or other organic disease" 4 . The 1990 American College of Rheumatology (ACR) criteria paper noted: "To avoid argument over the existence or nonexistence of 'secondary fibromyalgia,' which is believed by some investigators to be fibromyalgia caused [author's italics] by another condition, we adopted the term secondary-concomitant fibromyalgia [original italics] to indicate fibromyalgia occurring in the presence of another significant rheumatic disorder which may have been caused by or was merely associated with the patient's fibromyalgia"5. In the current paper by Wolfe, et al, the following definition is given: Secondary FM is FM occurring in the presence of another clinically important and dominant medical disorder ${ }^{3}$. We agree with this simplified definition and note that there is an ever-increasing list of disorders that have been associated with FM [Table 1; plus the same table with references (Supplementary Table 1, available with the online version of this article)]; whether a patient is designated as having primary FM becomes dependent upon a diligent search for all such associated comorbidities.

In 1 study, only $13 \%$ of patients with a 1990 ACR criteria diagnosis of FM lacked another pain disorder ${ }^{6}$; however, that study did not record other potential comorbidities such as sleep apnea, migraine, temporomandibular disorder, restless leg syndrome, endometriosis, irritable bowel syndrome, overactive bladder syndrome, etc. Thus, it is quite likely that
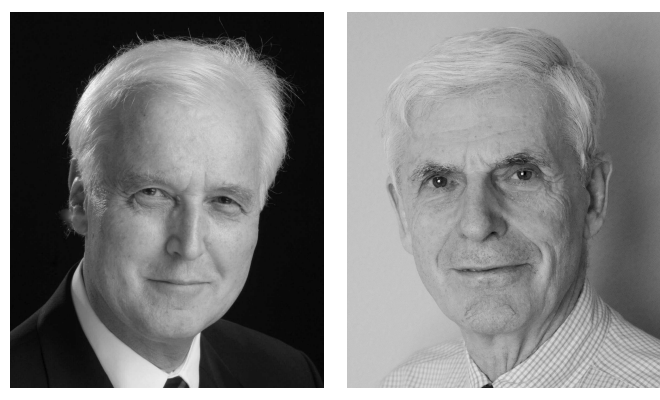

the prevalence of primary FM is negligible if all comorbidities are accounted for. Similar observations have led some to question the existence of primary $\mathrm{FM}^{7}$.

Wolfe, et al conclude that primary and secondary FM are equivalent regarding symptom burden ${ }^{3}$. This is the same conclusion reached in the 1990 ACR criteria study ${ }^{5}$. The difference between the 2 studies is the use of the polysymptomatic distress (PSD) scale as an analyzing tool. Whether the 2 groups are equivalent regarding symptom burden is critically dependent upon which symptoms are available for analysis. The current study used data from the National Data Bank for Rheumatic Diseases (NDB); this restricts the number of rheumatic diagnoses used in the analysis, for as is noted, "Another potential limitation to these data was our inability to know about other diagnoses." Because it is not possible to extract a comprehensive list of FM comorbidities from the NDB, the authors limited their analysis to patients with rheumatoid arthritis (RA) and FM (2016 criteria). The first set contained 1525 participants with a diagnosis of FM without evidence of a concomitant inflammatory disorder, but FM patients with noninflammatory disorders would be included in the study. Thus, one might expect the "primary" FM group to include patients with disorders such as migraine, osteoarthritis, endometriosis, postsurgical pain, peripheral neuropathy, etc.; in other words, it would not be a "clean" group. The second set contained 12,037 patients with RA, with $22.3 \%$ having comorbid FM (as per the 2016 criteria). No information is given as to whether any of the patients with RA had significant comorbidities other than FM. Thus, this study compared the clinical features of a group of patients with FM, who may or may not have had significant comorbidities, with 2 groups of patients with RA, those with and without comorbid FM. Further, both arms of the rheumatoid group could have had other significant comorbidities. Bearing in mind the rarity of primary FM as noted above, the conclusions reached in this paper should be questioned.

See Primary and secondary fibromyalgia, page 204

Personal non-commercial use only. The Journal of Rheumatology Copyright @ 2019 . All rights reserved. 
Table 1. Fibromyalgia comorbidities.

\begin{tabular}{|c|c|c|}
\hline Pain-related & Non-pain-related & Associated Syndromes \\
\hline TMJ disorder & Chemical sensitivity & Irritable bowel syndrome \\
\hline Low back pain & Thermal sensitivity & Interstitial cystitis \\
\hline Migraine headaches & Light sensitivity & Restless leg syndrome \\
\hline Tension headaches & Auditory sensitivity & Chronic fatigue syndrome \\
\hline Hypermobility syndrome & Mitral valve prolapse & \\
\hline Pelvic pain disorder & Hyperparathyroidism & \\
\hline Dyspareunia & Paresthesia & \\
\hline Muscle cramps & Rhinitis & \\
\hline Myofascial syndromes & Urticaria & \\
\hline Abdominal pain & Mood disorders & \\
\hline Endometriosis & Suicidal ideation & \\
\hline Mastalgia & Poor balance & \\
\hline Osteoarthritis & Functional dyspepsia & \\
\hline Rheumatoid arthritis & Seizures & \\
\hline Ankylosing spondylitis & Strokes & \\
\hline SLE & Diabetes & \\
\hline Sjögren syndrome & Celiac disease & \\
\hline Psoriasis & Gluten sensitivity & \\
\hline Carpal tunnel & Obesity & \\
\hline Multiple sclerosis & Autoimmune thyroid disease & \\
\hline Crohn disease & HIV infection & \\
\hline Ulcerative colitis & Primary immunodeficiency & \\
\hline Fragile $\mathrm{X}$ spectrum disorder & Hypothyroidism & \\
\hline Coronary heart disease & Hepatitis & \\
\hline
\end{tabular}

All listed comorbidities have at least 2 supporting references in Supplementary Table 1, available with the online version of this article. TMJ: temporomandibular joint; SLE: systemic lupus erythematosus; HIV: human immunodeficiency virus.

The authors remarked on the importance of their findings: "the demonstration of the presence and equality of FM and FM symptoms across medical conditions provides a reason to doubt much FM research in which FM is treated as disease to be compared with other diseases. Such studies are common, but are innately defective and invalid, and lead to erroneous conclusions because fibromyalgia can also be present in what would be considered the control group" 3 . The problem with this statement is that the authors have not demonstrated the presence and equality of FM symptoms across medical conditions. They have demonstrated that some FM symptoms are common across some medical conditions. However, these commonalities include fatigue, poor sleep, depressive symptoms, and intermittent bowel symptoms, which are part of the 2016 criteria and are some of the commonest symptoms seen in primary care ${ }^{8}$. An important issue is the noninclusion of some common noninflammatory disorders; for example, migraine headaches occur in about $30 \%$ of patients with $\mathrm{FM}^{9}$ and diabetes is reported in about $18 \%{ }^{10}$.

By "universality" of the PSD, it is implied that almost all chronic conditions can be located on the PSD spectrum and compared on a common basis of symptom severity. The claim for the universality is based on demonstrating that RA and FM do not differ on the PSD and associated symptoms when the PSD is statistically controlled for. There are 2 issues here. The first concerns the adequacy of the PSD as a representative measure of FM and symptom severity in general ${ }^{11}$. An important feature of any psychometric scale is how well the construct is defined and how representative it is of the construct that it purportedly measures. The PSD is narrow in content because $81 \%$ of the PSD is devoted to pain locations and fatigue/energy ( 25 of 31 points) $)^{12}$. It is therefore not surprising that RA/FM similarity is found, given that their symptoms are common to many chronic conditions (e.g., pain, fatigue, lack of energy, depressed mood). By contrast, the Symptom Impact Questionnaire (SIQR) ${ }^{13}$ contains more symptoms and can discriminate between FM, RA, and systemic lupus erythematosus, using the symptoms of "difficulty sitting for 45 minutes" and "tenderness to touch" 14 . Moreover, in a study to find the best symptoms that distinguish FM from other patients with chronic pain, the symptom of "persistent deep ache over most of the body" was far more discriminatory than a visual analog scale pain rating ${ }^{15}$. These SIQR questions are not used in the PSD.

The second concern is the use of a statistical control to demonstrate the equivalency of FM and RA on symptom burden. That the results showed equivalency between the 2 groups is not surprising: if the PSD is a primary determinant of RA/FM symptom differences, then controlling for the PSD may erase or attenuate differences on PSD and correlated outcomes. 
This article ${ }^{3}$ centers on the PSD scale as an organizing tool for making the argument that RA can be used as an ideal control group for comparative studies of pain in FM. The title, with its focus on primary and secondary FM, may be misleading because the actual analysis compares RA and FM, not primary and secondary FM. In the process of doing so, the article claims that the 2016 criteria have more than $90 \%$ accuracy, with a receiver-operation characteristic curve of 97\%. This statement may be misleading because it will be read to mean that the PSD has an extraordinary accuracy in identifying patients with FM. However, the $90 \%$ accuracy is generated from 2 indices derived from the same underlying PSD measure, in that the predictor variable is the PSD and the classification variable is the 2016 criteria, which uses the PSD in its diagnostic algorithm; hence they are 2 sides of the same coin and are not independent assessments. It is for this reason that a nearly perfect area under the curve of 0.97 was generated. In fact, the 2016 criteria identified only $53 \%$ of FM as defined by physicians contributing to the NDB.

Because of the increasing realization that FM has associations with many other disorders, the question of the equivalency of primary and secondary FM is no longer germane, as most patients with "primary" FM will be found to have comorbidities.

ROBERT M. BENNETT ${ }^{(1)}$, MD, FRCP, MACR, Oregon Health Sciences University, Portland, Oregon;

RONALD FRIEND (D), $\mathrm{PhD}$,

Stony Brook University, Stony Brook, New York, USA.

Address correspondence to Dr. R.M. Bennett, 6119 SW California St., Portland, Oregon 97219, USA. E-mail: bennetrob1@ comcast.net

\section{ONLINE SUPPLEMENT}

Supplementary material accompanies the online version of this article.

\section{REFERENCES}

1. Salaffi F, Gerardi MC, Atzeni F, Batticciotto A, Talotta R, Draghessi $\mathrm{A}$, et al. The influence of fibromyalgia on achieving remission in patients with long-standing rheumatoid arthritis. Rheumatol Int 2017;37:2035-42.
2. Zammurrad S, Munir W, Farooqi A. Disease activity score in rheumatoid arthritis with or without secondary fibromyalgia. J Coll Physicians Surg Pak 2013;23:413-7.

3. Wolfe F, Walitt B, Rasker JJ, Häuser W. Primary and secondary fibromyalgia are the same: the universality of polysymptomatic distress. J Rheumatol 2019;46:204-12.

4. Hench PK. Secondary fibrositis. Am J Med 1986;81:60-2.

5. Wolfe F, Smythe HA, Yunus MB, Bennett RM, Bombardier C, Goldenberg DL, et al. The American College of Rheumatology 1990 criteria for the classification of fibromyalgia. Report of the Multicenter Criteria Committee. Arthritis Rheum 1990;33:160-72.

6. Bennett RM, Friend R, Marcus D, Bernstein C, Han BK, Yachoui R, et al. Criteria for the diagnosis of fibromyalgia: validation of the modified 2010 preliminary American College of Rheumatology criteria and the development of alternative criteria. Arthritis Care Res 2014;66:1364-73.

7. Forslind K, Fredriksson E, Nived O. Does primary fibromyalgia exist? Br J Rheumatol 1990;29:368-70.

8. Kroenke K, Price RK. Symptoms in the community. Prevalence, classification, and psychiatric comorbidity. Arch Intern Med 1993; $153: 2474-80$

9. Giamberardino MA, Affaitati G, Martelletti P, Tana C, Negro A, Lapenna D, et al. Impact of migraine on fibromyalgia symptoms. J Headache Pain 2015;17:28.

10. Yanmaz MN, Mert M, Korkmaz M. The prevalence of fibromyalgia syndrome in a group of patients with diabetes mellitus. Rheumatol Int 2012;32:871-4.

11. Friend R, Bennett RM. A critical examination of the polysymptomatic distress scale construct as a symptom severity questionnaire. J Rheumatol 2015;42:1364-7.

12. Friend R, Bennett RM. Evaluating disease severity in chronic pain patients with and without fibromyalgia: A comparison of the symptom impact questionnaire (SIQR) and the polysymptomatic distress scale (PSD). J Rheumatol 2015;42:2404-11.

13. Bennett RM, Friend R, Jones KD, Ward R, Han BK, Ross RL. The revised fibromyalgia impact questionnaire (FIQR): validation and psychometric properties. Arthritis Res Ther 2009;11:R120.

14. Friend R, Bennett RM. Distinguishing fibromyalgia from rheumatoid arthritis and systemic lupus in clinical questionnaires: an analysis of the revised Fibromyalgia Impact Questionnaire (FIQR) and its variant, the Symptom Impact Questionnaire (SIQR), along with pain locations. Arthritis Res Ther 2011;13:R58.

15. Jones KD, Aebischer JH, St John AW, Friend R, Bennett RM. A simple screening test to recognize fibromyalgia in primary care patients with chronic pain. J Eval Clin Pract 2018;24:173-9.

J Rheumatol 2019;46:127-9; doi:10.3899/jrheum.180611 